In the same time period as this study, 1719 patients underwent colonoscopy and biopsy due to unexplained diarrhoea.

Conclusions These data confirm that BAM is common but is under-investigated in comparison with microscopic colitis. Although it is not associated with any 'hallmark' symptoms, it is readily identified by experienced clinicians as suggested by the high pick up rate, presumably on the basis of clinical acumen. It occurs in approximately $2 \%$ of patients following cholecystectomy, although the true prevalence in this population may be greater. These data also support the BSG guideline audit recommendation of SeHCAT in IBS-D as a quality improvement target.

\section{PWE-083 PREVALENCE OF OPIOID THERAPY IN PATIENTS WITH FUNCTIONAL GASTROINTESTINAL DISORDERS REVIEWED AT A TERTIARY CENTRE}

${ }^{1,2}$ Amir Mari*, ${ }^{2}$ Marcella Pesce, ${ }^{2}$ Kalp Patel, ${ }^{2}$ Fotios Lezgidis, ${ }^{2}$ Anton Emmanuel, Natalia Zarate-Lopez. ${ }^{1}$ Nazareth Hospital Emms Trust, Nazareth, Israel; ${ }^{2}$ Gl Physiology Unit, UCLH, London, UK

\subsection{6/gutjnl-2019-BSGAbstracts.403}

Introduction The appropriateness of chronic opioid use in patients with non-cancer pain is increasingly challenged due to limited evidence of its efficacy and the risk of problematic use and dependence. The side effects of opioids on gut function suggest this therapy should be particularly avoided in patients with functional gastrointestinal (GI) disorders. Data on the prevalence of opioid use in patients with functional GI disorders is unknown. We aimed to evaluate the prevalence of opioid use in functional GI patients referred to a tertiary Neurogastroenterology clinic and to determine the risk factors for their initiation.

Methods 100 consecutive outpatients (85 females, 37 \pm 15 years) with functional GI disorders referred for a specialist opinion at our tertiary referral centre were retrospectively reviewed. Two independent researchers (AM and FL) carried out a detailed chart evaluation of the presence and type of comorbidities and concomitant pharmacotherapy. Patients were also specifically asked about opioid use, the duration of therapy and the clinical indication for starting the opioids.

Results Twenty-one patients (20F, 38 \pm 12 years) were taking chronic opioid therapy. The main reason for opioid initiation was chronic musculoskeletal pain (12 patients, 57\%), chronic abdominal pain (5 patients, 24\%) and chronic pelvic pain (1, $5 \%)$. The mean duration of the opioid treatment was $2.7 \pm 1$ years. The risk factors associated with opioids use are depicted in table 1 . In terms of management, patients under opioid were offered naloxegol (20\%), psychology-based interventions (29\%), and referral to pain specialists to rationalize opioid use $(38 \%)$. The majority of the patients in the opioid group $(80 \%)$ were referred for tertiary opinion from other areas of the UK, as compared to the $60 \%$ of patients without chronic opioid use.

Conclusions Female patients with chronic functional GI disorders with associated extra-intestinal functional pain syndromes and psychological comorbidity are prescribed opioid therapy more frequently. These patients are at increased risk of developing eating difficulties requiring advanced nutritional support, which is known to be more complicated in opioid users. Access to multidisciplinary coordinated therapy including pain management and psychological therapy is required early in these patients to avoid these adverse outcomes.

\begin{tabular}{llll}
\multicolumn{1}{l}{ Abstract PWE-083 Table 1} & \\
\hline & $\begin{array}{l}\text { Opioids } \\
\text { (n.21) }\end{array}$ & $\begin{array}{l}\text { Non-opioids } \\
\text { (n.79) }\end{array}$ & $\mathrm{p}$ value \\
\hline Female/Male & $20 / 1(95 \%)$ & $66 / 13(84,6 \%)$ & $\mathrm{ns}$ \\
Age (mean \pm SD) & $36,5 \pm 16$ & $38,3 \pm 12$ & $\mathrm{~ns}$ \\
$\begin{array}{l}\text { Hypermobility Elhers- } \\
\text { Danlos }\end{array}$ & $11(52 \%)$ & $14(18 \%)$ & $\mathrm{p}<0.001$ \\
Depression & $4(38 \%)$ & $3(16,6 \%)$ & $\mathrm{p}<0.05$ \\
Artificial nutrition & $17(19 \%)$ & $32(5 \%)$ & $\mathrm{p}<0.05$ \\
\hline
\end{tabular}

\section{PWE-084 ANORECTAL PHYSIOLOGY PROFILES IN YOUNG ADULTS, MIDDLE-AGED ADULTS AND OLDER ADULTS WITH FAECAL INCONTINENCE}

Ismail Miah*. Guy's and St Thomas' Nhs Trust, Greater London, UK

\subsection{6/gutjnl-2019-BSGAbstracts.404}

Introduction Adult patients of all age report faecal incontinence (FI) symptoms but little has been done on comparing the anorectal physiology profiles in young adults (YA), middleaged adults (MAA) and older adults (OA). This study investigates the topic in question.

Method All patients selected for this study complained of FI and underwent anorectal physiology investigation that was performed by an experienced gastrointestinal physiologist between October 2010 and October 2013. Any patients with history of constipation or anorectal surgery were excluded. YA, MAA and OA patients were grouped into ages 1-0 years, >4-5 years and $>65$ years based on Erik Erikson's theory of adult development.

Patients' FI symptoms were sub-classified based on urge faecal incontinence (UFI) and passive faecal incontinence (PFI) symptoms. The anorectal physiology tests included manometry assessment of anal sphincter motor function [1], rectal balloon sensory to distension study [2] and anorectal mucosal electrical stimulation to assess for sensory deficit [3]. Each test were benchmarked against normal range [4]. Appropriate 3-group ANOVA and $\chi 2$ statistical tests were used to compare the anorectal physiology profiles in YA, MAA and OA.

Results The female: male ratio for YA, MAA and OA were respectively 59:10, 174:34 and 100:28.

YA showed significantly higher incidence in weak involuntary EAS contraction compared to MMA and OA patients $(\chi 2=6.0, p=0.0498)$. This was consistent with the clinical presentation as $74.2 \%$ YA patients only report UFI symptoms and UFI symptoms were less prevalence in MAA (57.1\%) and OA $(33.6 \%)(\mathrm{p}<0.00001)$.

In contrast to YA, PFI symptoms were more frequently reported by MMA and OA which can be explained by the combination of higher prevalence of hypotensive anal tone $(\chi 2=32.6, \quad \mathrm{p}<0.00001)$ and diminished sensory to rectal capacity volume $(\chi 2=82.0, \mathrm{p}<0.00001)$ observed in MMA and OA. This pathophysiology in the anorectal compliance found in MMA and OA would increase the risk of developing passive rectal overflow incontinence that patient's report as PFI symptoms. Furthermore, MAA and OA patients also have higher incidences of anal sensory deficit compared to YA $(\chi 2=10.4, p=0.0056)$ suggesting of unfelt anal seepage that is also perceived as PFI. 
Conclusion Distinctive FI symptomatology and sensorimotor pathophysiology profiles has been identified in young and older adults. YA have higher prevalence of UFI symptoms owing to EAS dysfunction. MAA and OA showed higher prevalence in PFI symptoms through combination of diminished sensory of the rectal reservoir filling, reduced anal tone to prevent rectal outflow of faecal matter and blunted anal sensory making them susceptible to faecal seepage.

\section{REFERENCE}

1. . Rogers J., et al. 1989. Br. J. Surg. 76(6): p60-09

2. . Farthing M. and Lennard-Jones J., 1978. Gut. 19: p6-9

3. . Roe A., 1986. Br. J. Surg. 73(4. ): p31-12

4. . Jameson J., et al., 1994, Br J Surg. 81(11):p1689-92.

\section{PWE-085 THE CLINICAL VALUE OF PERFORMING COUGH ASSESSMENT IN PATIENTS WITH FAECAL INCONTINENCE DURING ANAL MANOMETRY}

Ismail Miah. Guy's and St Thomas' Nhs Trust, Greater London, UK

\subsection{6/gutjnl-2019-BSGAbstracts.405}

Introduction The external anal sphincter (EAS) involuntary contraction invoked by cough mechanism is usually abandoned during anal manometry studies because its clinical value remains unknown. In this study, we retrospectively review the EAS contractions invoked by cough in patients undergone biofeedback therapy (BFT) for faecal incontinence.

Method Patients were selected between October 2010 and October 2013. Each patient had:

Anal manometry test performed by an experienced gastrointestinal physiologist

Attended at least two biofeedback therapy (BFT) sessions

Patients with constipation symptoms leading to overflow incontinence, flatus/mucus incontinence without faeces or having a rectal prolapse protruding externally via anus on visual inspection were excluded.

Anal manometry was performed using 8-channel water-perfused radial catheter in accordance to [1]. Anal tone and EAS contractions (induced by voluntary manoeuvre and involuntary via cough mechanism) were performed twice at the high pressure zone of the anal canal and the highest contraction amplitude were recorded. These physiological parameters were benchmarked against normal range [2]. Statistical $\chi 2$, t-test, odd ratio (OR) and positive predictive value (PPV) were employed.

Results Patient gender female: male demography was 97:18 (aged between 2-0 years). None of the patients showed or experienced faecal incontinence episodes during the cough manoeuvre.

In this cohort of patients, $29.5 \%$ demonstrated normal voluntary EAS contraction and $68.7 \%$ demonstrated a normal EAS involuntary contraction $(p<0.0001)$. This suggests the two EAS function assessment are different and the voluntary EAS contraction did not represent the full potential of the EAS contraction.

Patients who responded to BFT showed significantly increased EAS involuntary contraction $(77.4 \mathrm{cmH} 2 \mathrm{O}$ vs $43.7 \mathrm{cmH} 2 \mathrm{O}, \mathrm{p}<0.00001)$. Sensitivity to responding BFT when normal involuntary EAS contraction was observed is $72 \%$ and specificity to not responding to BFT when involuntary EAS contraction amplitude was hypotensive is $53.3 \% \quad(p=0.0242)$. When a normal EAS involuntary contraction is demonstrated during manometry, the odds of patients responding to BFT nearly triples and good prediction to responding to BFT was observed $(\mathrm{OR}=2.96$, PPV=91.1\%).

Conclusion This study shows the clinical value of performing EAS involuntary contraction invoked by cough as a diagnostic parameter that differs from the voluntary EAS assessment. EAS involuntary invoked by cough may also be a clinical predictor for BFT outcome.

\section{REFERENCE}

1. Rogers J., et al. 1989. Br. J. Surg. 76(6): p60-09

2.. Jameson J., et al., 1994, Br J Surg. 81(11):p1689-92.

\section{PWE-086 SUBTYPES OF FAECAL INCONTINENCE}

Ismail Miah*. Guy's and St Thomas' Nhs Trust, Greater London, UK

\subsection{6/gutjnl-2019-BSGAbstracts.406}

Introduction Urge faecal incontinence (UFI) and passive faecal incontinence (PFI) are forms of anal incontinences described by patients that are not treated differently nor received subtype classification for treatment. This study addresses the pathophysiology of faecal incontinence subtypes.

Method Patients were selected based on UFI and PFI symptoms who also underwent various anorectal physiology studies according to protocols [1]. They were performed by an experienced clinical gastrointestinal physiologist.

All patients had normal endo-anal ultrasound screening and the rectum criteria includes patients with incontinences of mixed subtypes, mucus and flatus incontinence without stools, constipation with overflow incontinence and rectal prolapse detected protruding externally from the anus on visual inspection.

Appropriate $\chi^{2}$ and t-test were employed to assess the patient demography and anorectal physiology profiles.

Results Total number of patients selected is 304: UFI group ( $\mathrm{F}: \mathrm{M}=181: 35$, aged 2-6years) and PFI group ( $\mathrm{F}: \mathrm{M}=66: 22$, aged 2-1 years).

Patients with PFI are generally older $(\mathrm{p}<0.00001)$ and men are more likely to complain of PFI than UFI (25\% vs $16.2 \%$, $\mathrm{p}=0.0374$ ).

Patients with UFI symptoms tend to open their bowels more frequently compared to patients with PFI symptoms (4.0 vs $2.8, \mathrm{p}=0.0005)$. Their stool consistency to bowel opening (including UFI episodes) are generally formed (Bristol Stool Chart [BSC] types -) and patients with PFI generally pass stools (including incontinent) that are loose/liquid motion (BSC types -$)(\mathrm{p}<0.00001)$.

There was no statistical difference in the functional anal length calculated from the manometric pull through $(\mathrm{p}=0.2610)$.

In patients with PFI symptoms, significantly reduced anal resting pressure was observed $(p=0.0001)$ and higher incidence of hypotensive anal tone was observed $(65 \%$ vs $42 \%$, $\mathrm{p}=0.0002$ ).

Patients with UFI symptoms, demonstrated significantly lower voluntary peak pressure $(p=0.002)$, involuntary cough contraction $(\mathrm{p}=0.011)$ and endurance squeeze pressures $(p=0.015)$. Respectively, their hypotensive prevalence were significantly higher: $43.1 \%$ vs $30 \% \quad(\mathrm{p}=0.0196), 43 \%$ vs $31 \%$ $(\mathrm{p}=0.0383)$ and $83.4 \%$ vs $71 \%(\mathrm{p}=0.0088)$.

The rectal sensory function to distension were generally normal in both subtypes of FI and there was no statistical 